\title{
Local and Global Election Result collation \& Transmission System
}

\author{
Ismaila L. Enegi ${ }^{1 *}$ Steve A. Adeshina ${ }^{2 *} \&$ Adegboyega Ojo ${ }^{3 *}$ \\ ${ }^{1}$ Department of Computer Science, Nile University of Nigeria, Abuja, \\ E-mail : ismailukman@gmail.com \\ ${ }^{2}$ Department of Computer Engineering, Nile University of Nigeria, Abuja \\ E-mail : steve.adeshina@nileuniiversity.edu.ng \\ ${ }^{3}$ National University of Ireland, Galway, Ireland
}

\begin{abstract}
We present a technique for election results collation and transmission in largely populated areas. In our earlier works we presented an electronic balloting and result transmission systems [1] [2]. Whereas these systems perform creditably well where voters are within estimated number of between 500 and 1000, a problem of local collation was introduced where the population exceeds 2000 where multiple devices have to be introduced. We achieved local collation by providing an admin device for result collation from each e-balloting devices at various voting points. The results collated at local level were further transmitted to central collation center from each polling units after local result have been announced. The system provided way to ensure local result display at individual Polling Units (a requirement of Nigerian laws) and reduces congestion during results' transmission.
\end{abstract}

Index Terms-Mobile Election, Electronic Voting, Wireless Voting, Smart phone wireless communication, E-Balloting

\section{INTRODUCTION}

Elections are democratic ways of choosing leaders who represent us. Elections have been around for a very long time. Elections in various countries have attracted a lot of controversies. This is because of the enormous power associated with the outcome of elections, the stakes are extremely high. Politicians have found, and are still finding ways of tilting the results of elections in their favor. Notwithstanding the manner of the conduct of elections, whether manual or automated, controversies continue to trail the outcome of elections. It is needless to say that, the manual process of voting is subject to the most irregularities. It also important to note that irregularities are often well thought out and they may occur at every stage of the electioneering process. These processes include, Registration, Authentication, Balloting \& Tabulation and results transmission. In most developed jurisdictions Registration and Identification are well developed and they serve as solid foundation for a good elections. This is often not true for developing nations like Nigeria. It is indeed clear that countries with developed infrastructures tend to require much less application of technology than the developed ones due to high trust level.

Electronic Voting have equally been around for several years. Whereas some countries are adopting e-voting technologies with huge success, others are abandoning technologies already adopted due to trust issue. Other countries continue to stick to the age long manual approach [3]. Situating electronic voting within the context of developing nations present several challenges. These challenges are not unconnected with the lack of infrastructure such as Internet penetration, power, mobile phone access and other mitigating factors which include literacy levels and difficult terrains.

It is upon this developing nations' background that this work is set. Indeed most of our early works in this domain seeks to find ways and means to engender good governance within a developing nations setting. Our e-Balloting system seeks to present a ballot paper on an Android tablet [2]. The simplicity and user friendliness attempt to help illiterate voters. Our Result Transmission System [1] seeks to enable transmission of results from remote areas where internet access may be non-existent. This work attempt to close the gap created by the elections transmission system. It is often the requirement of the law that results of elections be declared immediately at the voting points. This will pose no problems where a single tablet device is deployed to a Polling Station. However in urban areas we find voters in polling points are far in excess of the estimated capacity and more devices and personnel are often deployed to ensure voting closes as at when due. There is a need to locally collate these various results and present same as attributable to a specific Polling Units. This work therefore makes local and global transmission possible. It is indeed needless to say that the interregnum between the time of voting and publication of results provide opportunities to mischief makers who falsify elections results and change the outcome of elections.

Figure 1a shows a voting screen from the E-Balloting System [2], while Figure $1 \mathrm{~b}$ shows a tabulated result from a single device. Figure 1c shows a screen-shot from the result 
transmission system [1]. Suffice it to say here that these results are transmitted as Short Message Services (SMS). This ensures that results can be sent from areas where Internet access is not available.

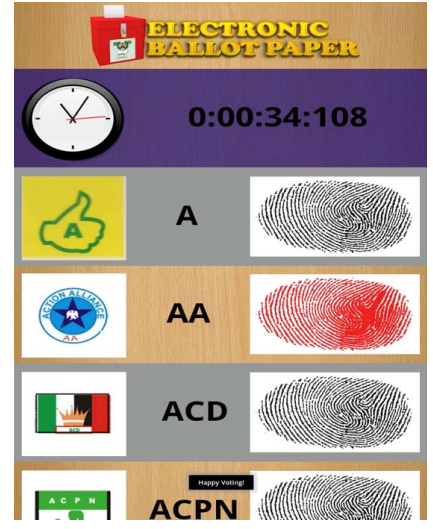

(a) Voting device as Eballoting tablet

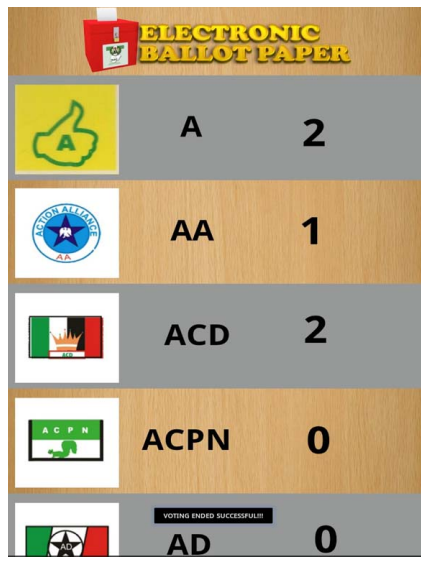

(b) Polling unit result display

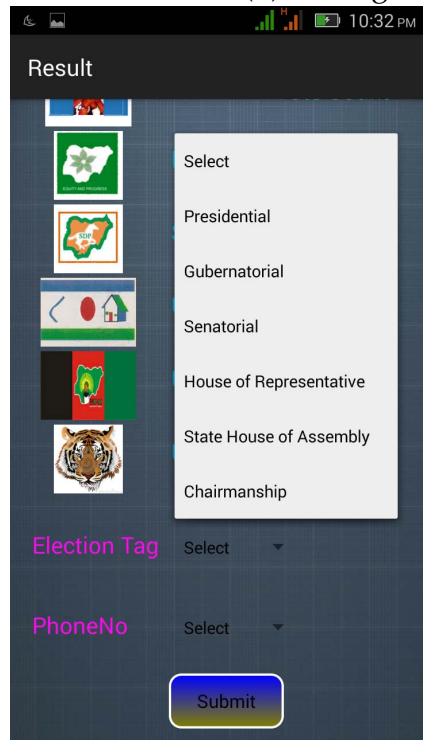

(c) Voting device for result transmission

Fig. 1: Screen shot from previous work

Figure 2 shows the process of a typical local collation at a Polling Unit. It must be noted that the same device that is used for Balloting also tabulates the results and forward same to the Admin device which then transmits the result to the Central server. The Figure illustrates four voting devices submitting their results to the admin device for onward transmission to the Central server.

\section{Typical Elections Result Collation at Polling Unit}

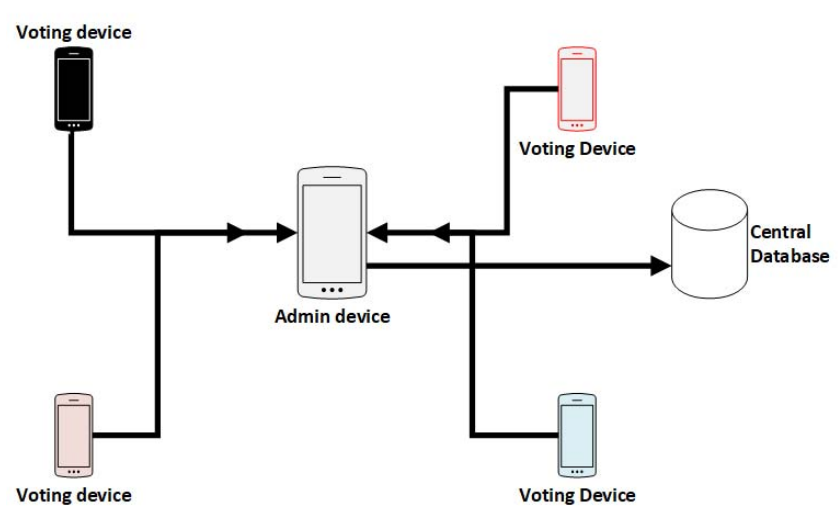

Fig. 2: Typical result collation at PU

The uniqueness of this work is that it provides a local collation of results before sending same to a central Server. This is often overlooked in most result transmission system. The consequence of the absence of a local collation is that it will be impossible to automatically declare a Polling Unit result without resorting to manual addition. In most electoral constituencies declaration of result at the polling point is mandatory. The manual addition is fraught with several irregularities. Whereas each operating device could sent its results to a central server where collation can take place, the approach will however further congest the already scarce bandwidth. We further enhanced our transmission system by completing the central transmission using JSON format, this approach provides effective data structure and improves error avoidance through mismatch. We are not aware of similar implementation in Literature.

The closest work to this work is that of Adeshina et al.where an automated result transmission was presented [1]. This work differs in that it allows for local collation. Additionally results were transmitted in raw SMS format whereas our results are being transmitted with JSON format so help reduce issues with formatting.

\section{Related Works}

Some related works are discussed in the following sections.

\subsection{Electronic Voting}

Wolchok et al. [4] present a security analysis of a real time Indian Electionic Voting Machine (EVM).They evaluated its security in light of relevant election procedures and concluded that despite tight security measures, they are still vulnerable to security attacks. This effort is very relevant to our work in order to ensure minimal possibility of vulnerability. Rajendiran et al. [5] implemented a secure Electronic voting machine using biometric fingerprint identification ,Aadhar card database was used for fingerprint identification. During the time of voting, the e-voting process authentication can be done using finger vein sensing, which enables the electronic ballot reset and permit voters to cast their votes. Also the resultant votes could be sent wirelessly to the nearby Database Administration unit through WIFI 
communication. Kohno et al. [6] did a security analysis of voting machines. Their analysis shows that voting systems are far below even the most minimal security standards applicable in other contexts. Through this analysis they identify several problems including unauthorized privilege escalation, incorrect use of cryptography, vulnerabilities to network threats, and poor software development processes.

\subsection{Wireless Voting}

Chakraborty et al. [7] designed an Electronic Voting machine used to count poll results during voting period and temporary stored for a later time when results will be transferred through a signal transmitter for the receiver of the counting module. This work is of high consideration because it adopted wireless and real-time result display during voting process. Ashish Ramteke et al. [8] proposed a voting machine which can be used to determine the eligibility of voter by scanning the eye pattern and also the vote count is not kept into the same machine itself instead it is stored in a remote server by converting it into radio waves. Here nobody can possibly increase the vote count (data) of machine. Even in case of damage or deliberate attack to voting machine there will not be any problem to continuity of the election process.

\subsection{Wireless communication in Smart phone}

Jiang Junjie [9] worked on Communication between Sensors and Mobile Phone involves mobile sensing of air pollution monitoring system for environment and individuals. Based on the cooperation between device and Smart phone the air pollution are measured and uploaded to database server for computing a visualized pollution level. The relevance of this work is to analyze the effectiveness of wireless communication with smart phones. Peer to Peer Communication between smart devices was also demonstrated by Atul R. Ghumare et al.. His work provide efficient display of file stored on a remote computer, saving image files and text files to the computer and handling control of a remote computer using a android smart phone [10]. Wenlong Shen [11] present working of device to device (D2D) communication, several attacks that face WiFi-Direct-based communications. Because pairwise key establishment lies in the area of securing D2D communications, they introduced a short authentication-string-based key agreement protocol and analyze its security performance, further more SASbased key agreement protocol was also integrated into the existing WiFi Direct protocol, the implementation of this work was demonstrated in an android smart phone.

\subsection{Mobile Election}

Mpekoa et al. [12] designed a mobile phone voting framework and an application. The mobile phone voting application facilitates users to spontaneously and timeously vote using existing mobile phone networks and technologies. Dipali More [13] Proposed a system that provides secure and efficient online vote casting and also paper ballot system if online voting fails. In their system, voting does not require access to the internet,but only required at the time of online registration only. All other processes were done offline through SMS messaging. Adeshina et al. [1] demonstrated the influence of manual intervention on free and fair election. In their work, Results transmission were made possible without the use of internet connection since SMS was used. The results were collated in central database and displayed in real-time.

\subsection{E-Balloting}

Adeshina et al. [2] presented a fully designed electronic balloting system, this system presents an electronic ballot on a tablet. This allows for ease of use. Patel et al.[13] presented various Electronic voting Method like voting by kiosk, Internet, telephone, punch card, and optical scan ballot. They also described the pron and cons of the presented voting method. Keerthika et al. [14] proposes the need of real-time electronic voting system in election implemented with a Voting kiosk.

\section{Methodology}

This research adopted agile software methodology because of the continuous check on the performance of the system for further improvement in newer versions. Our requirement analysis was performed from information obtained from informal Interviews, document inspections and observation. The direct involvement one of the authors in the Nigeria's Electoral Management Body also provided very deep insights.

\subsection{Software Requirement Analysis}

This system was designed and implemented a computer environment with sensitive requirement as listed bellow.

Minimum System Requirements

1) Smart Phone

- Android OS Support Api 15 to date

- RAM Memory $128 \mathrm{MB}$ and above

- Memory Disk 512MB and above

2) Wifi-Hotspot

3) WiFi communication

\subsubsection{System Process Diagram (Admin)}

This diagram shows step-wise procedure of voting, result collation and transmission of the admin device application. 
Local \& Global Election Result Collation Proprietary Device App (Process Diagram)

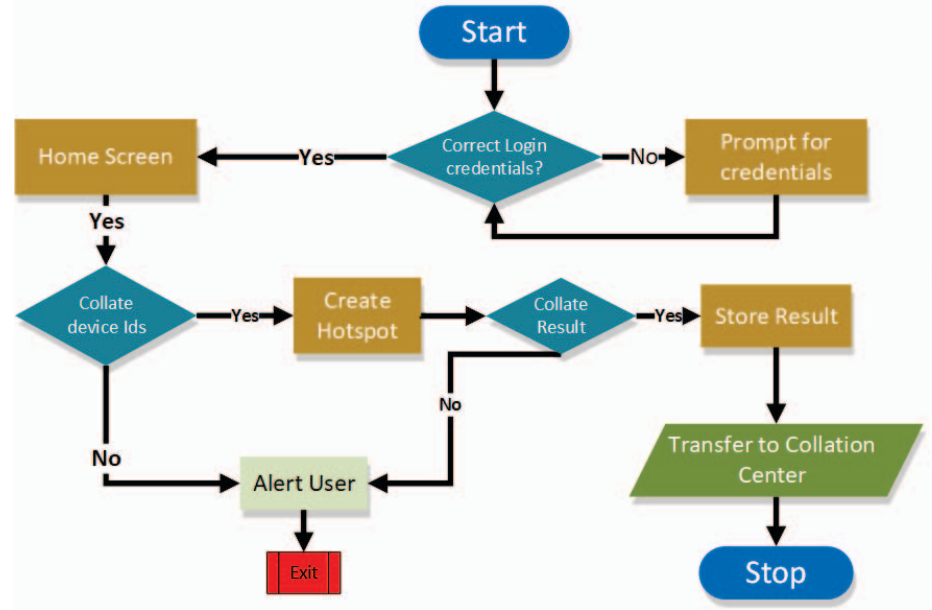

Fig. 3: Admin Device Process Diagram

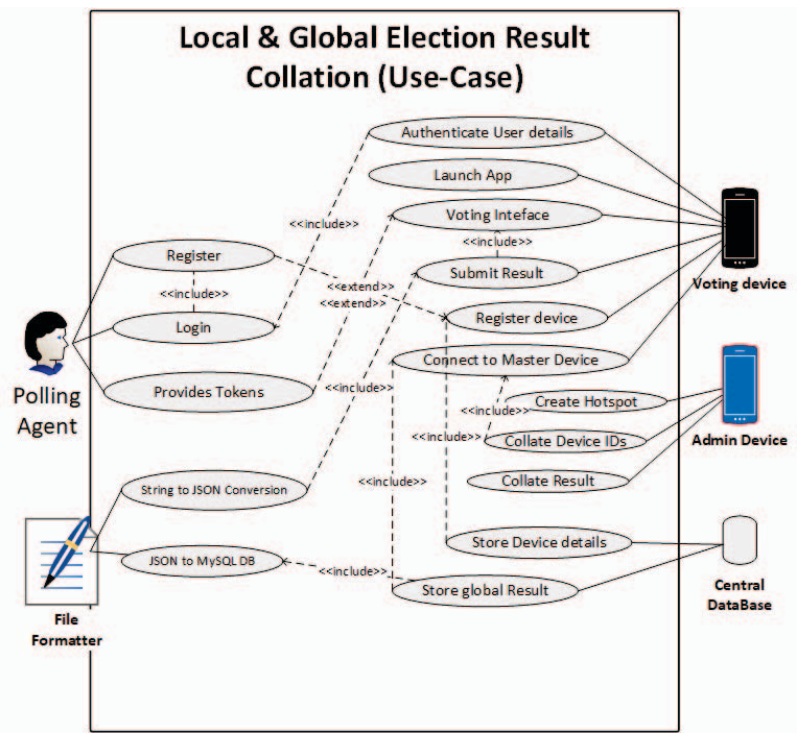

Fig. 5: System Use-case Diagram

\subsubsection{System Process Diagram}

This involves the processes of voting and result transfer to admin device. the process begins with the launch of eballot app and end after results are successfully submitted to admin device.

\section{Local \& Global Election Result}

\section{Collation Voting Device App (Process Diagram)}

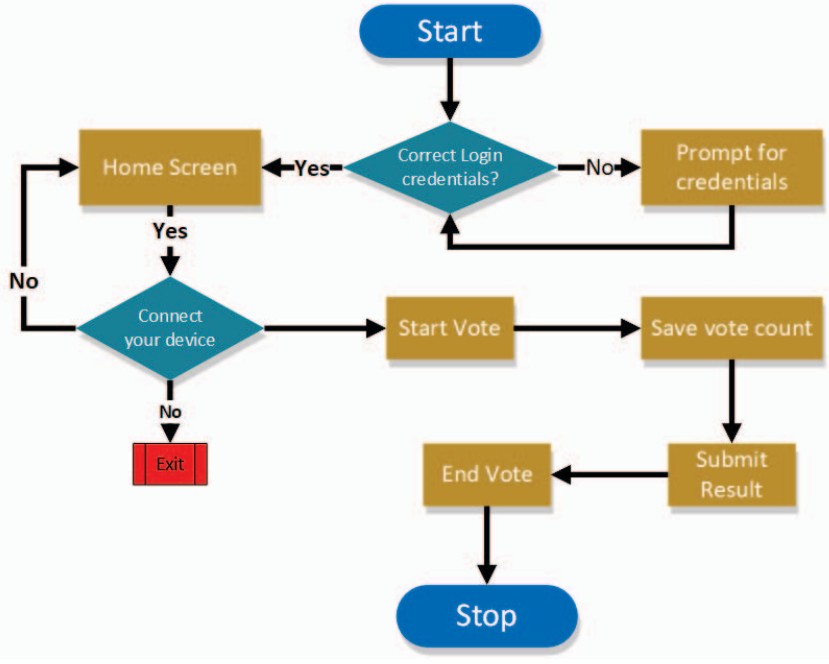

Fig. 4: System Process Diagram

\subsubsection{System Use-case Diagram}

This illustration shows an overview of interactions between various actors and other components of the system.

\section{SYSTEM IMPLEMENTATION}

WLAN Result Collation: This process involves election admin device to switch-on its wifi-hotspot for other devices to connect. This process is done when other voting tabs which have been used for voting exercise as E-ballot are ready to transfer their results for local result collation. When devices are connected result are sent through java sockets to the admin device for storage and local result display.

Voting Process Mechanism: This section involve the use of voting tablet as E-balloting devices. This basically provide a software simulation of manual ballot paper. The vote count are automated and done as voting continue These results are then formatted into JSON objects by our implementation of a java object JSONObject.java which parses results text from a String or a JSONTokener to produce a map-like object of our party-vote count values, results are further stored in an SQL-Lite database for easy retrieval after the voting process is concluded, results are transferred to admin devices over wifi communication.

Result Collation Mechanism: Every device at a voting point is expected to send its result through a wireless communication to an admin device at a polling unit in order to avoid congestion and ensure result declaration at polling unit level, this is a requirement in Nigeria and some other countries in Africa. Our results are collated into SQL database both locally and globally, for local collation, android sqlite is used as the suitable database in android platform and MySQL is used in global data storage. our data format is preserved throughout the process of transmission and storage. JSON data format is used when result are transmitted and SQL format is used for data storage. 


\section{Input \& Output Screens}
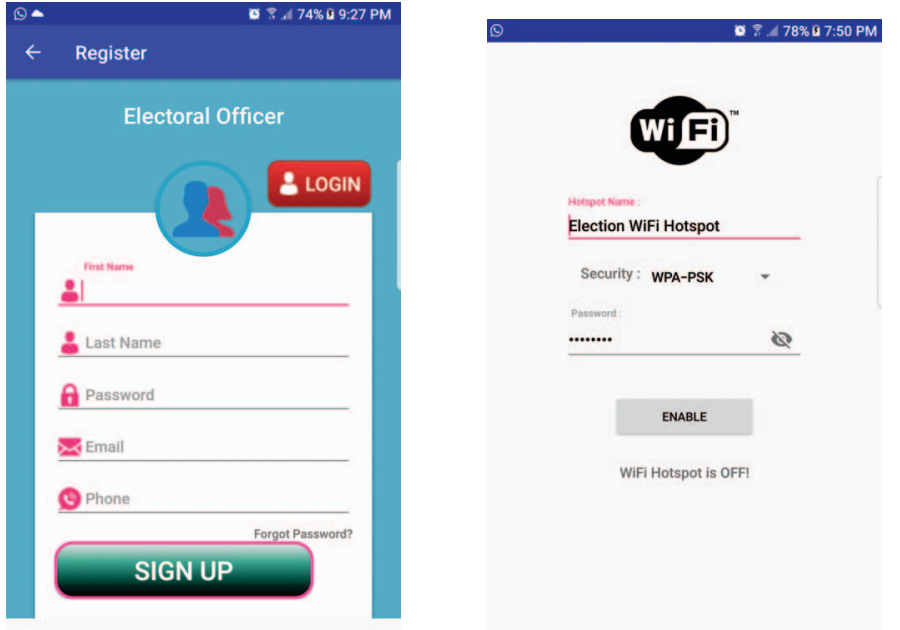

(a) Polling unit agent registration

(b) Admin device hotspot

Fig. 6: Device logging page and wifi hot-spot creation

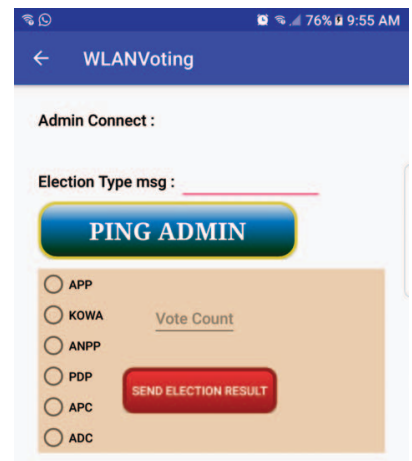

(a) Voting device send result to admin device

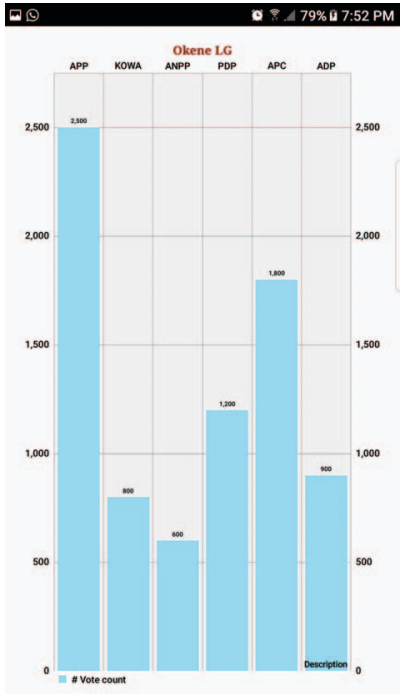

(b) chart result display
Fig. 7: Result transmission \& display

\subsection{Results Analysis}

This test was carried out to demonstrate system ability to resist error introduction. The accuracy of the system was also well examined to ensure vote counts cannot be altered either in transit or in storage. Data is collated by providing this system to 30 users for testing and evaluation. The results are later calculated by considering the users choices and parameters being measured (accuracy and error introduction). Suffice it to say here that the designed system was used for the Student Union elections in Nile University of Nigeria. Results obtained were far better when compared with manual collations.

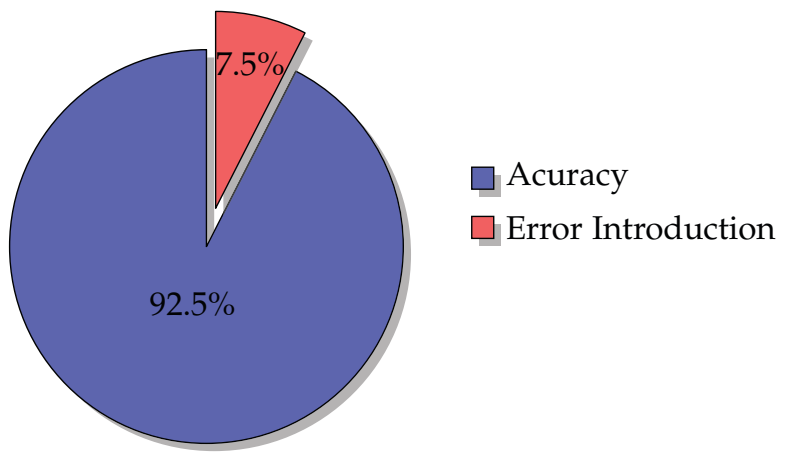

Fig. 8: Pie chart of system validity test

\section{Discussion \& Conclusion}

This work demonstrates the need for local collation of election results in densely populated areas where several devices may have to be deployed. The importance of early declaration of results cannot be over emphasized, since this is the interval when election results are altered and elections compromised. This work assumes lack of Internet access, though it is able to operate equally well where connectivity exist. The environmental context for this work is Nigeria where all the regulatory and technical framework to transmit result using an Android based mobile device have been concluded. The Independent National Electoral Commission (INEC) seem to be introducing e-Voting in phases, where the Registration, Authentication processes have been automated. Plans to automate the Result Collation process have been concluded using some of the methods we described in this work. It is obvious that the balloting process has yet to be automated for obvious reasons of trust of the electorate. It is hope that the INEC will pilot the proposed electronic balloting in urban areas where the issues of local collation of results will definitely arise. Even if the INEC fail to pilot these method during the forth coming elections, it is almost definite that the populace will expect a full blown e-voting in subsequent elections. Though Nigeria has been used as a case study in this work. The methods are applicable to similar contexts where illiterate is high, where there is a huge digital divide, where the terrain is difficult and where elections is a do or die affair.

Additionally, we are not aware of any other works in this domain in the literature. Whereas there are many esoteric works on mobile and internet voting from uncontrolled environment like our homes, the approaches are unlikely to be adopted due to the number of people with access and due to the fact that there is no way to ensure that the mobile device or computer system is free of malicious software.

In further works, we hope to tighten security by providing end-to-end encryption of data in transit. This can be implemented using any of the available mobile friendly data encryption algorithms. This work currently retrieve device location during voting process. In order to provide better security GPS location should also be considered during the time of result transmission and details of transfer can be stored in database as well. Additional security on results can be considered using block-chain technology. 


\section{REFERENCES}

[1] S. Adeshina, I. E. Lukman, and K. Ibrahim, "Mobile Based Election Result collation \& transmission System," International Journal of Computing Techniques, vol. 4. No.1, pp. 56-63, 2017.

[2] S. Adeshina, I. E. Lukman, and A. Ojo, "E-balloting System," International Journal of Computing Techniques, vol. 4. No.1, pp. 7986, 2017.

[3] S. Adeshina and A. Ojo, "Factors for e-voting adoption- analysis of general election in nigeria," Elsevier: Government Information Quarterly, vol. Article in Press, 2017. [Online]. Available: http://dx.doi.org/10.1016/j.gi1.2017.09.006

[4] W. Scott, H. K. P. Arun, and R. Gonggrijp, "Security Analysis of Indias Electronic Voting Machines," Proceedings of the 17th ACM conference on Computer and communications security - CCS '10, vol. 4, no. 02, pp. 1013-1015, 2015.

[5] R. S., I. R., I. S., and S. M., "Aadhar Based Electronic Voting System and Providing authentication on Internet of Things," International research journal in Advance Engineering and Technology (IRJAET), vol. 3, no. 2, pp. 1918-1925, 2017.

[6] T. Kohno, A. Stubblefield, A. D. Rubin, and D. S. Wallach, “Analysis of an Electronic Voting System," IEEE Computer Society Press, no. May, p. 23, 2004.

[7] subhadeep Chakraborty, S. Karmakar, R. Jana, and S. Dey, “Design of secure wireless real-time voting machine," International Journal of Innovative Research in Electrical, Instrumentation and Control Engineering, vol. 3. Issue 9, pp. 1-21.

[8] R. Ashish and D. V. Rojatkar, "Global wireless e-voting," International Journal of Electrical and Electronics Research, pp. 201-204.

[9] J. Jiang and V. Sivaraman, "School of Engineering and Communication between Sensors and Mobile Phone," Thesis Report, no. 3297513, 2011.

[10] A. R. Ghumare, N. D. Patil, C. D. Holkar, and V. D. Badgujar, "Peer to Peer Communication between Android Device and PC and Video Surveillance using Android Device," pp. 175-180.

[11] W. Shen, B. Yin, X. Cao, L. X. Cai, and Y. Cheng, "Secure device-todevice communications over WiFi direct," IEEE Network, vol. 30, no. 5, pp. 4-9, 2016.

[12] N. Mpekoa, "Designing, developing and testing a mobile phone voting system in the South African context Noluntu," Proceedings of the 8th International Development Informatics Association Conference, pp. 372-385.

[13] D. More, S. Mehzabin, M. Awaskar, S. Ghongde, S. Wattamwar, and D. Tadpelliwar, "Mobile Voting System," International Journal of Emerging Technology and Advanced Engineering, vol. 5:9001, pp. $1-5$.

[14] G. M. Keerthika and S. A. S. Hemalatha, "Smart Wireless Voting System using Smart Card Kathir College of Engineering Coimbatore 62," International Journal for Scientific Research \& Development-, vol. 4 issue 02, no. 02, pp. 1013-1015, 2016. 